\title{
Preconcentration in Gas or Liquid Phases Using Adsorbent Thin Films
}

\author{
Antonio Pereira Nascimento Filho ${ }^{\mathrm{a}}$, Alexsander Tressino de Carvalho ${ }^{\mathrm{a}}$, \\ Maria Lúcia Pereira da Silva ${ }^{\mathrm{a} *}$, Nicole Raymond Demarquette ${ }^{\mathrm{b}}$

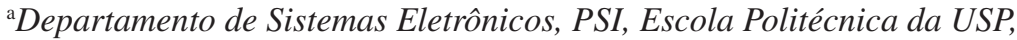 \\ Av. Prof. Luciano Gualberto, 158, room A1-46, 05508-900 São Paulo - SP, Brazil \\ ${ }^{\mathrm{b}}$ Departamento de Eng. de Materiais, PMT, Escola Politécnica da USP, \\ 05508-900 São Paulo - SP, Brazil
}

Received: December 2, 2004; Revised: July 13, 2005

\begin{abstract}
The possibility of preconcentration on microchannels for organic compounds in gas or liquid phases was evaluated. Microstructures with different geometries were mechanically machined using poly(methyl methacrylate) - PMMA as substrates and some cavities were covered with cellulose. The surfaces of the microchannels were modified by plasma deposition of hydrophilic or hydrophobic films using 2-propanol and hexamethyldisilazane (HMDS), respectively. Double layers of HMDS + 2-propanol were also used. Adsorption characterization was made by Quartz Crystal Measurements (QCM) technique using reactants in a large polarity range that showed the adsorption ability of the structures depends more on the films used than on the capillary phenomena. Cellulose modified by double layer film showed a high retention capacity for all gaseous compounds tested. However, structures without plasma deposition showed low retention capacity. Microchannels modified with double layers or 2-propanol plasma films showed higher retention than non-modified ones on gas or liquid phase.
\end{abstract}

Keywords: preconcentration, microchannels, microstructure and plasma polymerization

\section{Introduction}

Sample preconcentration and removal of interference from sample are key procedures in sample pretreatment for micro total analysis system ( $\mu$ TAS) production ${ }^{1}$. One of the main advantages of micro-size preconcentrator is the possibility of a larger preconcentration efficiency, sometimes larger than the one found on macro systems. These procedures can be achieved using several methods and adsorption is a key parameter for many of them but up to now it has not been properly explored. Likewise, adsorbent films may be of great use for sensor development but have received little interest. Recently, one small analysis equipment was developed by Sandia Labs ${ }^{2,3}$. The system could be tailored for gas or liquid phase analysis, using chromatography ${ }^{4}$ or electrophoresis ${ }^{5}$, respectively. One of the fundamental steps of this device was the sample collection and concentration, i.e. the preconcentrator unit. In that case, the preconcentration was done by an adsorbent film deposited on a silicon nitride membrane and desorption occurred by heating of this membrane. Another important step was the detection, which used an array of surface acoustic wave (SAW) sensors recovered by an adsorbent film. The same way of SAW sensors, chemiresistor sensors may use adsorbent films. There exist report of chemiresistor and SAW sensors that were tested for VOC's (volatile organic compounds) detection on soil and groundwater ${ }^{6}$ showing good results for m-xylene, but not for TCE (trichloroethylene), which required preconcentration $^{7-10}$.

VOC's have harmful environmental effects and are commonly analyzed using gas chromatography and conventional preconcentrators ${ }^{11}$, i.e. gaseous phase. These preconcentrators essentially consist of one big stainless steel pipe, full of an adsorbent product. Recently, some novelties in small sorbent trap, named microtrap ${ }^{12-14}$ and miniaturized systems ${ }^{15}$ have been reported. A microconcentrator, which can also be used as injector for gas chromatography, was produced at the surface of a silicon wafer using a thin polymeric layer for adsorption process. Another study reported the development of a multistage micro-preconcentrator for volatile and semi-volatile organic compounds for indoor VOC detection. The miniaturized device consists of "roughly spherical granules, similar to $200 \mu \mathrm{m}$ in diameter, of a high surface area, graphitized carbon"16-20. The detection limit can be as low as $25 \mathrm{ppb}$ due to the surface area of the adsorbent material. These small and miniaturized systems usually use planar geometries ${ }^{21}$ to avoid difficulties for quick heating and cooling that would lead to large desorption time ${ }^{22}$.

For liquid phases, adsorption is useful for many techniques, such as voltametry ${ }^{23}$ or capillary electrophoresis ${ }^{24}$ and the detection ranges from ions $\mathrm{s}^{25}$ to proteins ${ }^{26}$. Solid phase extraction is another method that has achieved some miniaturization and could use adsorbent films. Also chromatographic columns can be miniaturized and it is another example of where adsorbent films are needed. In aqueous matrix, semi-volatile organic compounds have been analyzed using membrane extraction ${ }^{27}$ and liquid chromatography. All of them also request the use of microchannels.

Recently microchannels were produced using non-conventional processes, such as ink jet systems ${ }^{28}$. Some microchannels with nonplanar structure ${ }^{29}$ were produced using ordinary machining tools and sealed without heating. This approach presented the following advantages: it was possible to produce a low cost structure with interchangeable parts that can be easily dismantled any time; this structure could receive any type of film deposited by dry or wet processes as no high temperature bonding step was requested in its processing. The structure was similar to a screw with a microthread and a cylinder to encapsulate it. Inlet and outlet systems were made with stainless steel capillaries and the sealing of all structure occurs only on the edges using silicone glue. 
Microchannels favor capillary phenomena and it was already used to preconcentrate VOC from nitrogen using only capillarity effects ${ }^{30}$. One way to avoid capillarity effects is the use of cavities that would favor mainly adsorption. Cavities are similar to the adsorption area on sensors ${ }^{30}$.

In our former works two adsorbent films were obtained using a $40 \mathrm{KHz}$ planar plasma reactor and 2-propanol and hexamethyldisilazane (HMDS) as reactants. For 2-propanol plasma polymerization ${ }^{31}$, it was shown that the polar film obtained is a selective membrane impermeable to water but permeable to organic compounds. The polar films obtained also presented another interesting characteristic when deposited on hydrophobic substrates once a discontinued layer was obtained. This discontinued layer was called island and allowed the increase of the adsorption surface area. HMDS plasma films ${ }^{32}$ show interesting characteristics, such as selective permeation of alcohol in aqueous solutions. Moreover non-planar microchannels covered with HMDS films showed good adsorption properties for organic compounds on $\operatorname{air}^{29}$ and cavities also showed some facilities for testing several different substrates ${ }^{33}$.

Therefore, the aim of this work is as follows: 1 ) to verify the possibility of production of the adsorbents 2-propanol plasma polymerized films in other frequencies and geometry than the $40 \mathrm{KHz}$ planar reactor formerly used; and 2) To evaluate the use of microchannels and cavities for preconcentration of organic compounds in gas or liquid phases using these films.

\section{Materials and Methods}

\subsection{Substrates and reagents}

All chemical reagents used were p.a. (Casa Americana Ltd., Brazil). The substrates used were: 1) type p, $<100\rangle, 10-20 \Omega . \mathrm{cm}$, 3 inch diameter silicon wafers (Silicon Sence, Inc., USA) for film characterization; 2) poly(methyl methacrylate) - acrylic - $5 \mathrm{~mm}$ thickness bars (Plasttotal Ltd., Brazil) for microstructures manufacturing; 3) piezoelectric quartz crystal (PQC), 4.096 MHz, $11.0 \mathrm{~mm}$ diameter, $0.8 \mathrm{~mm}$ thickness, Ag electrode (Hosonic Industrial Ltd, Brazil) to determine the adsorption and permeation characteristic of the films; 4) cellulose film (Comercial Baitaca Papéis e Embalagens Ltd., Brazil), $20 \mu \mathrm{m}$ thickness, for adsorption tests in cavities.

\subsection{Plasma deposition}

The plasma deposited films were obtained in a home made planar $^{31}$ or tubular reactors ${ }^{34}$. The depositions were performed at room temperature and the flow of reagent admitted to the reactor was of the order of $1.0 \mathrm{~g} / \mathrm{min}$. The planar reactor is a capacitive plasma reactor, that can be coupled to a $40 \mathrm{kHz}$ or $13,56 \mathrm{MHz}$ power supply. It consists of a stainless steel reaction chamber with two $20.0 \mathrm{~cm}$ diameter electrodes, $3.0 \mathrm{~cm}$ apart from each other. The sample is normally placed on the grounded electrode. Base pressures of 20 mTorr can be reached within a few minutes and the reagents admitted pure or by carrier gas. The working pressures and input power were chosen to range from 0.5 Torr to 2.0 Torr and from $30 \mathrm{~W}$ to $200 \mathrm{~W}$, respectively. These values allowed a long mean free path for ions and atoms and medium energy for active species. The tubular reactor is also a capacitive plasma reactor that can be coupled to a $13.56 \mathrm{MHz}$ power supply. The reaction chamber is made of glass, has a diameter of $20.0 \mathrm{~cm}$ and a length of $100 \mathrm{~cm}$. The sample is normally positioned in the center region of the chamber to avoid non-uniformity of the deposited films. Base pressures of 20.0 mTorr can be reached within a few minutes and the reagents can be introduced pure or by carrier gas. The working pressures and input power were chosen to range from 0.3 Torr to 0.6 Torr and from $150 \mathrm{~W}$ to $300 \mathrm{~W}$, respectively.

\subsection{Characterization}

The films were analyzed using the following techniques: a) profilometry using a Dektak 3030 profilemeter (Veeco Instruments Inc, USA) to determine their thicknesses; b) FTIR using a FTS-40 (BioRad Laboratories, Hercules, CA, USA) to determine their chemical structure; and c) contact angle measurements using a "Rame-Hart" goniometer and drops of water or organic aqueous solutions. The films were also analyzed by optical and atomic force microscopy using a microscope model Nanoscope E (Digital, Inc., CA, USA) and Secondary Electron Microscopy model SEM 515 (Philips, USA).

Quartz crystal measurements (QCM) were used to analyze adsorption on the deposited films. The contaminants tested were organic compounds carried by nitrogen (gas phase). QCM was also used to evaluate the retention or preconcentration on the structures on liquid phase. The contaminants used were aqueous solution of 2-propanol (50\% in vol.), acetone (50\% in vol.), acetophenone (saturated) and n-hexane (saturated) and water as eluent.

For QCM experiments, a homemade apparatus was used as described in a previous work ${ }^{31}$. A schematic diagram of the experimental setup for tests in gas phase and liquid phase are shown in Figures 1 and 2, respectively. The oscillator circuit of this system is based on a TTL hex-inverter integrated circuit 74VHCU04N (National Semiconductor, Santa Clara, CA). A microcomputer-interfaced HP 3458A multimeter (Hewlett Packart, Palo Alto, CA) was used in the frequency counter mode with $1 \mathrm{~Hz}$ resolution and data acquisition rate of 55 points $/ \mathrm{min}$. The pump used for liquid phase measurements is a pneumatic one operating from 0.5 to $5 \mathrm{~mL} / \mathrm{min}^{35}$.

\subsection{Structures manufacturing}

Two types of structures were machined using Computer Numeric Control-CNC (Protomat C100/HF, LPKF Laser \& Electronics AG, Germany) and acrylic substrates: cavities and microchannels. The cavi-

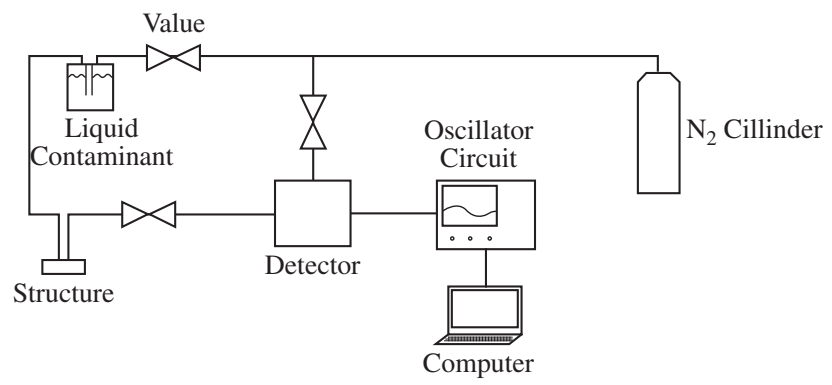

Figure 1. Schematic diagram for the experimental setup used for QCM in gas phase. The meaning of structure is microchannel or cavity.

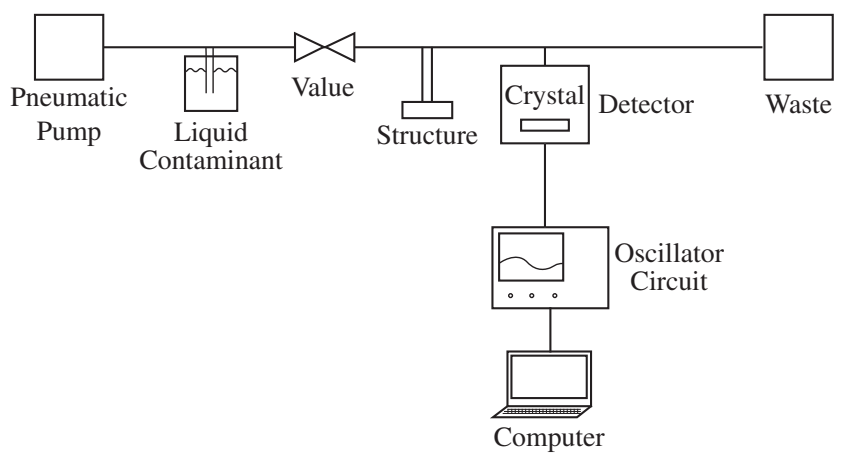

Figure 2. Schematic diagram for the experimental setup used for QCM liquid phase. 
ties consisted basically of a circular reduction of $100 \mu \mathrm{m}$ height and diameter of $2 \mathrm{~cm}$ on the substrate. The sealing was made using silicone glue. Another acrylic substrate was used to seal and add the inlet/outlet system. Some of these cavities received cellulose inside. The substrate was attached to the bottom of the cavity also using silicone glue. Three types of microchannels were made: a) chicane (500 $\mu \mathrm{m}$ depth, $1.0 \mathrm{~mm}$ width and $4.0 \mathrm{~cm}$ length $)^{36}$; b) spiral shape $(200 \mu \mathrm{m}$ depth, $500 \mu \mathrm{m}$ width and $40 \mathrm{~cm}$ length $)^{30}$; and c) non-planar shape $(100 \mu \mathrm{m}$ depth, $100 \mu \mathrm{m}$ width and $40 \mathrm{~cm}$ length $)^{29}$. The microchannels were made of acrylic and sealed using chloroform. Tubes were attached to the inlet/outlet systems (external diameter of 1/16 inch). All schematics of the structures are shown in Figures $3 \mathrm{a}, 3 \mathrm{~b}, 3 \mathrm{c}$ and $3 \mathrm{~d}$.

For some samples the surface of the structures was modified by deposition of a conformal thin film obtained by plasma polymerization using a capacitive plasma reactor coupled with a $40 \mathrm{kHz}$ power supply. The nature of the films deposited as well as the conditions of deposition are reported in Table 1.

\section{Results and Discussion}

This paper is divided in two sessions: 1) Main characteristics of the deposited films will be shown; and 2) they will be tested for preconcentration on microstructures.

\subsection{Production of plasma polymerized polar thin films}

\subsubsection{Results}

Table 2 summarizes the most important results obtained for plasma deposition of all the reactants used in the planar reactor coupled with either $40 \mathrm{KHz}$ or $13.56 \mathrm{MHz}$ power supplies. The obtained films showed similar chemical composition and contact angle measurements, however the deposition rates obtained are always higher using $40 \mathrm{KHz}$ than $13.56 \mathrm{MHz}$. Therefore, the use of $13.56 \mathrm{MHz}$ frequency did not favor polymerization and only acetone leads to film formation. Also, although it was possible to obtain stable plasma in

Table 1. Condition process for plasma deposition of adsorbent films on microchannels.

\begin{tabular}{clllll}
\hline Shape & \multicolumn{1}{c}{ Chicane } & \multicolumn{1}{c}{ Spiral } & \multicolumn{1}{c}{ Non-planar } \\
\hline \multirow{2}{*}{ Film } & 2-propanol & HMDS & Double layer* HMDS/2-propanol & 2-propanol & Ethyl ether \\
& $(1.0$ Torr, $150 \mathrm{~W}$, & $(0.5$ Torr, $50 \mathrm{~W}$, & $(0.5$ Torr, $50 \mathrm{~W}, 6000 \AA$ / & $(1.0$ Torr, $150 \mathrm{~W}$, & $(1.3 \mathrm{Torr}, 200 \mathrm{~W}$, \\
& $5200 \AA)$ & $6000 \AA)$ & 1.0 Torr, $150 \mathrm{~W}, 5000 \AA)$ & $5000 \AA)$ & $1000 \AA)$ \\
\hline
\end{tabular}

*Double layer means a first deposition of HMDS plasma film followed by a second one of 2-propanol plasma film. In these conditions a non-continuous film is obtained (island formation).

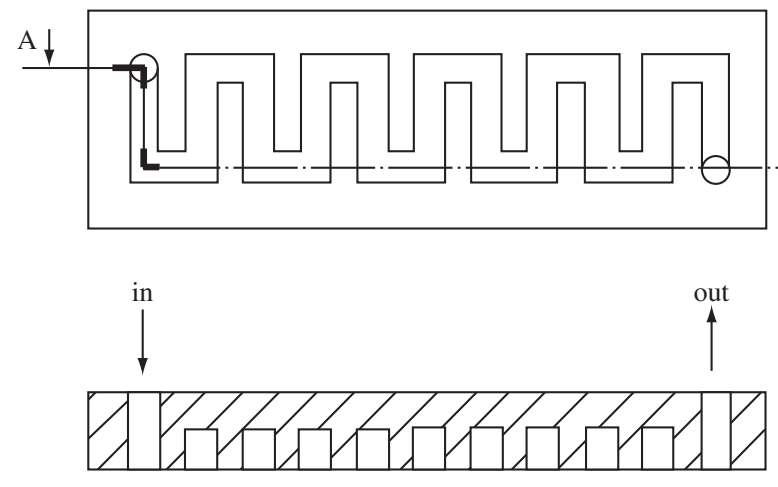

(a)

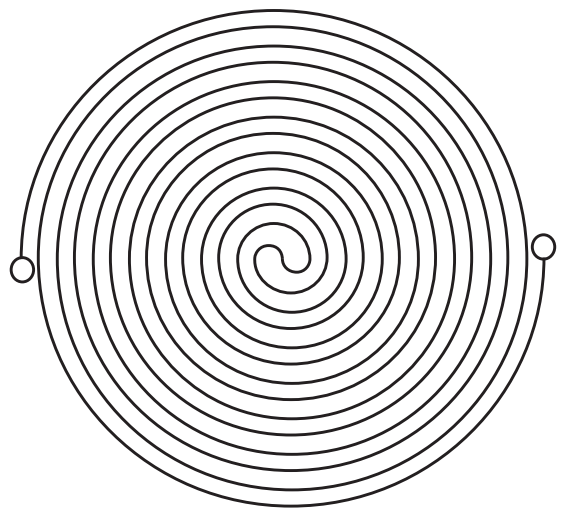

(b)

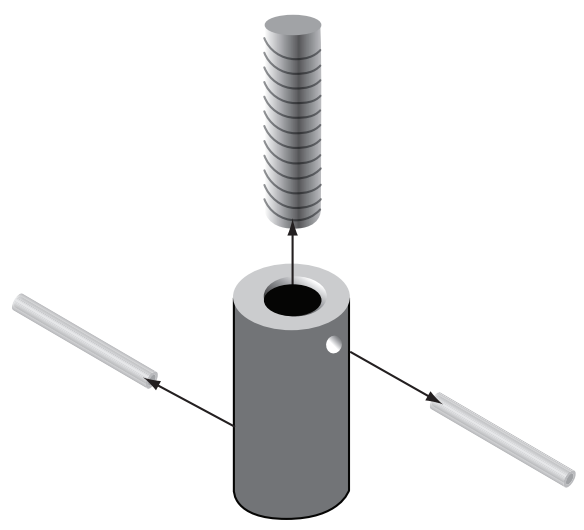

(c)
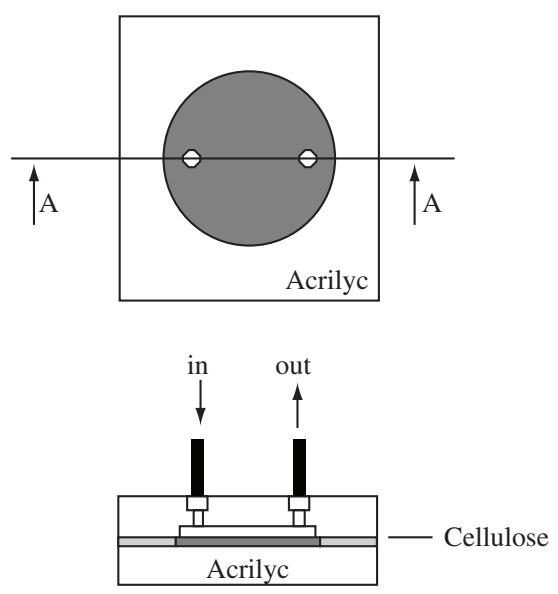

(d)

Figure 3. Sketch of the structures used: microchannels (a) chicane; b) spiral; and c) non-planar shape) and d) cavity. The letters "A-A" mean different view on the figures. 
any condition described in Materials and Methods using ethyl ether, the results showed that the chamber pressure increases less than $20 \%$ when the power supply is on, indicating that a small amount of active species has been formed. Thus, ethyl ether probably requests high energy density for active species formation at that frequency. Similar results were obtained for 2-propanol reactant. The use of a $13.56 \mathrm{MHz}$ power supply probably result in a different polymerization mechanism due to a low electrode bombardment with active species that leads to a small deposition rate.

The species detected by FTIR are mainly $\mathrm{CH}_{n}, \mathrm{OH}$ and $\mathrm{CO}$. For all films it was possible to find $\mathrm{OH}$ and/or $\mathrm{CO}$ species. However, the ratio between these polar species and $\mathrm{CH}_{\mathrm{n}}$ is quite different on these films. The high amount of $\mathrm{OH}$ on 2-propanol films is expected due to the presence of $\mathrm{OH}$ in the reactant structure. However, the high amount on acetone films is mainly due to reduction of the $\mathrm{CO}$ from acetone molecule. Likewise, the $\mathrm{CO}$ found in ether films is most likely due to oxidation. The similar contact angle measurements obtained for all films is due to polar species interaction between reactants and surface, such as hydrogen bond formation. Therefore, in these films the adsorption of polar species is possible, as showed by QCM.

The acetone plasma polymerized film obtained using the planar reactor coupled with the $13.56 \mathrm{MHz}$ power supply was deposited on PQC and characterized by QCM in gas phase. Figure 4 shows crystal frequency variation vs. time for different contaminants when exposed to vapors for 5 minutes.

It can be seen from Figure 4 a quick variation on frequency signal when the contaminants are inserted in the cell, except for 2-propanol. The difference of interaction between 2-propanol is probably due to hydrogen bridge formation. This result is similar to the ones found to ethyl ether polymerized and 2-propanol using $40 \mathrm{KHz}$.
Table 3 summarizes the most important results obtained for plasma deposition of all the reactants using the tubular reactor coupled with the $13.56 \mathrm{MHz}$ power supply. Figure 5 shows a typical infrared spectrum of a 2-propanol plasma polymerized film on this frequency. It can be seen from Table 3 that only 2-propanol leads to film formation but there is a low amount of polar species on this film. The high contact angle measurements, for water and 2-propanol aqueous solution, also indicate the formation a less polar structure, much more similar to a polyethylene structure.

Tests of island formation with double layers films of 2-propanol/ HMDS (i.e. 2-propanol plasma films deposited on HMDS plasma

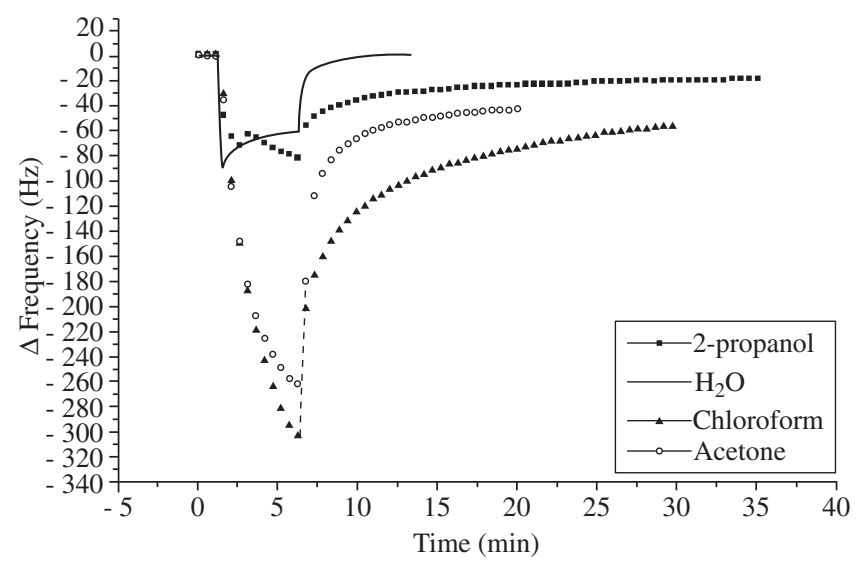

Figure 4. Crystal frequency variation vs. time for different contaminants. Acetone film deposited on PQC.

Table 2. Deposition conditions and most important characteristics obtained for plasma deposition of ethyl ether, 2-propanol and acetone on silicon substrates using planar reactor powered with $40 \mathrm{KHz}$ or $13.56 \mathrm{MHz}$.

\begin{tabular}{|c|c|c|c|c|c|c|}
\hline \multirow{3}{*}{$\begin{array}{l}\text { Properties } \\
\text { Power Supply }\end{array}$} & \multicolumn{6}{|c|}{ Reactants } \\
\hline & \multicolumn{2}{|c|}{ Ethyl Ether } & \multicolumn{2}{|c|}{ 2-propanol } & \multicolumn{2}{|c|}{ Acetone } \\
\hline & $40 \mathrm{kHz}^{29}$ & $13.56 \mathrm{MHz}$ & $40 \mathrm{kHz}^{31}$ & $13.56 \mathrm{MHz}$ & $40 \mathrm{kHz}$ & $13.56 \mathrm{MHz}$ \\
\hline Deposition rate $(\AA / \mathrm{min})$ & 372 & $\begin{array}{l}\text { No film } \\
\text { formation }\end{array}$ & 350 & $\begin{array}{c}\text { No film } \\
\text { formation }\end{array}$ & 500 & 20 \\
\hline Contact angle with $\mathrm{H}_{2} \mathrm{O}\left(^{\circ}\right)$ & 71 & - & 70 & - & 60 & 75 \\
\hline $\begin{array}{l}\text { Contact angle with } \\
\text { 2-propanol aqueous solution } \\
50 \% \text { vol. }\left({ }^{\circ}\right)\end{array}$ & 17 & - & 20 & - & 10 & - \\
\hline $\begin{array}{l}\text { Adsorption } \\
\text { characteristics (QCM) }\end{array}$ & $\begin{array}{l}\text { Adsorption of } \\
\text { polar species }\end{array}$ & - & $\begin{array}{l}\text { Adsorption of } \\
\text { polar species }\end{array}$ & - & $\begin{array}{l}\text { Adsorption of } \\
\text { polar species }\end{array}$ & $\begin{array}{l}\text { Adsorption of } \\
\text { polar species }\end{array}$ \\
\hline Main polar $\mathrm{CO} / \mathrm{CHn}(\%)$ & 36.7 & - & 10 & - & 30 & - \\
\hline $\mathrm{OH} / \mathrm{CHn}(\%)$ & 13.1 & - & 80 & - & 50 & 50 \\
\hline
\end{tabular}

Table 3. Deposition conditions and most important characteristics obtained for plasma deposition of ethyl ether, 2-propanol and acetone in silicon substrates using tubular reactor powered with $13.56 \mathrm{MHz}$.

\begin{tabular}{|c|c|c|c|c|}
\hline \multirow{2}{*}{\multicolumn{2}{|c|}{ Properties }} & \multicolumn{3}{|c|}{ Reactants } \\
\hline & & Ethyl Ether & 2-propanol & Acetone \\
\hline \multicolumn{2}{|c|}{ Deposition rate $(\AA / \mathrm{min})$} & No film formation & 150 & No film formation \\
\hline \multicolumn{2}{|c|}{ Contact angle with $\mathrm{H}_{2} \mathrm{O}\left(^{\circ}\right)$} & - & 80 & - \\
\hline \multicolumn{2}{|c|}{ Contact angle with 2-propanol aqueous solution $50 \%$ vol. $\left({ }^{\circ}\right)$} & - & 30 & - \\
\hline \multirow[t]{2}{*}{ Main polar species } & $\mathrm{CO} / \mathrm{CHn}(\%)$ & - & - & - \\
\hline & $\mathrm{OH} / \mathrm{CHn}(\%)$ & - & 5 & - \\
\hline
\end{tabular}


films) and acetone/HMDS obtained at $13.56 \mathrm{MHz}$ were attempted. The island formation and the wrinkling of the film were evaluated by optical microscopy. Chemical structure of the double layers was analyzed by infrared spectroscopy. Table 4 summarizes the results for island formation and Figure 6 shows typical infrared spectra of the film obtained.

As can be seen on Table 4, there is no island formation or wrinkling of the film. Therefore, the chemical mechanism of film deposition is different from the one occurring at $40 \mathrm{kHz}$ most likely due to the lower bombardment on $13.56 \mathrm{MHz}$, since higher bombardment allows the dangling bonds formation on the surface that favors reactions.

The infrared spectrum shown in Figure 6a presents all the species from HMDS and acetone, indicating it is possible to deposit acetone

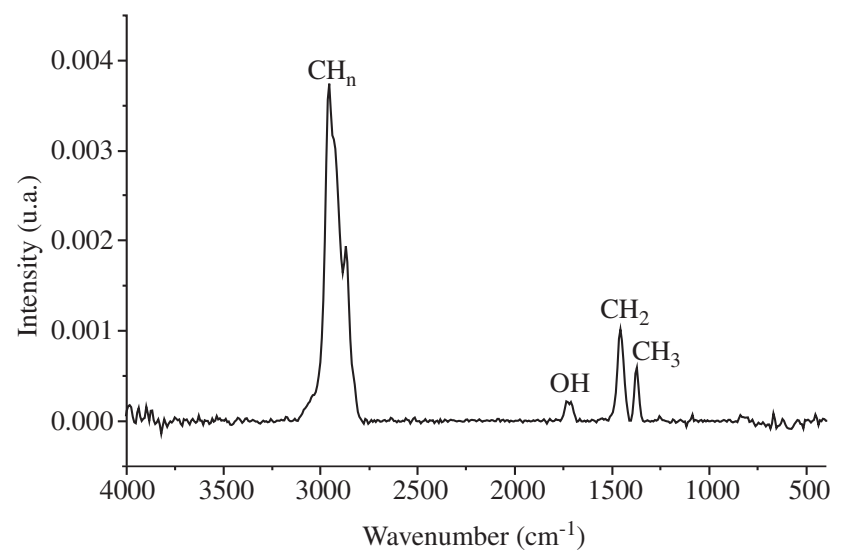

Figure 5. Typical FTIR spectrum of 2-propanol film (0.5 Torr; $400 \mathrm{~W}$; $200 \AA$ ) obtained using a tubular reactor coupled with a $13.56 \mathrm{MHz}$ as power supply.

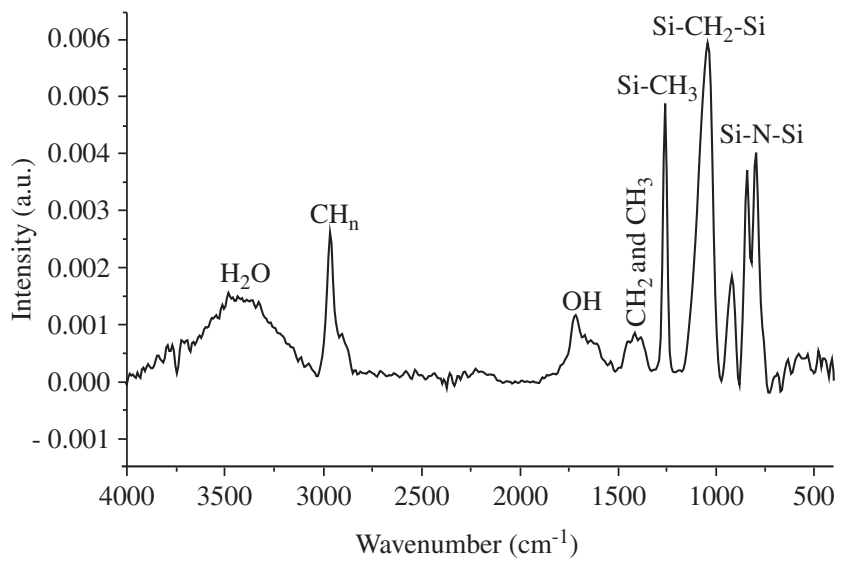

(a) on HMDS. It can also be seen from Figure 6a that a) both $\mathrm{CH}_{2}$ $\left(1450 \mathrm{~cm}^{-1}\right)$ and $\mathrm{CH}_{3}\left(1370 \mathrm{~cm}^{-1}\right)$ adsorption bands are not completely separated, showing that the acetone film may present a lot of defects, such as dangling bonds and that; and b) the $\mathrm{OH}$ band $\left(1700 \mathrm{~cm}^{-1}\right)$ is split due to $\mathrm{OH}$ present on the film structure and water trapped in the film, as evidenced by the $\mathrm{OH}$ band $\left(3400 \mathrm{~cm}^{-1}\right)$. Similar results were obtained for double layers 2-propanol plasma deposition. These spectra are also similar to the ones obtained for 2-propanol double layers produced at $40 \mathrm{KHz}$, as can be seen in Figure $6 \mathrm{~b}$.

The results presented above indicated that only the deposition of a double layer of 2-propanol/HMDS using the planar reactor coupled with the $40 \mathrm{kHz}$ power supply resulted in formation of islands. Then, these films were analyzed to determine the size of these islands, which can be tailored, as illustrated in Figure 7, which presents the structure of the films observed by SEM, AFM and optical microscopy. The main parameter that defines island size is the deposition time. The advantage of using different island size is the variation of surface area with smaller island presenting bigger surface area. In this work the double layers films deposited on cavities or microchannels were made according to the Figure 7a, i.e., with a big surface area. In these conditions the thickness of the island is approximately $50 \mathrm{~nm}$ and the increase of the surface area compared to the deposition without island formation is approximately 2 times.

Since double layers showed bigger surface area, adsorption tests were carried out using QCM analysis. Typical results can be seen in Figure 8. Double layer film showed an intermediate behavior between the one that was observed for HMDS films ${ }^{32}$ and 2-propanol films ${ }^{31}$ indicating that the double layers films are able to adsorb both polar and non-polar compounds.

\subsubsection{Discussion}

The results reported in this work as well as the ones obtained in previous works ${ }^{29,31,32}$ demonstrate the importance of the frequency power supply for the polar characteristics of organics films deposited

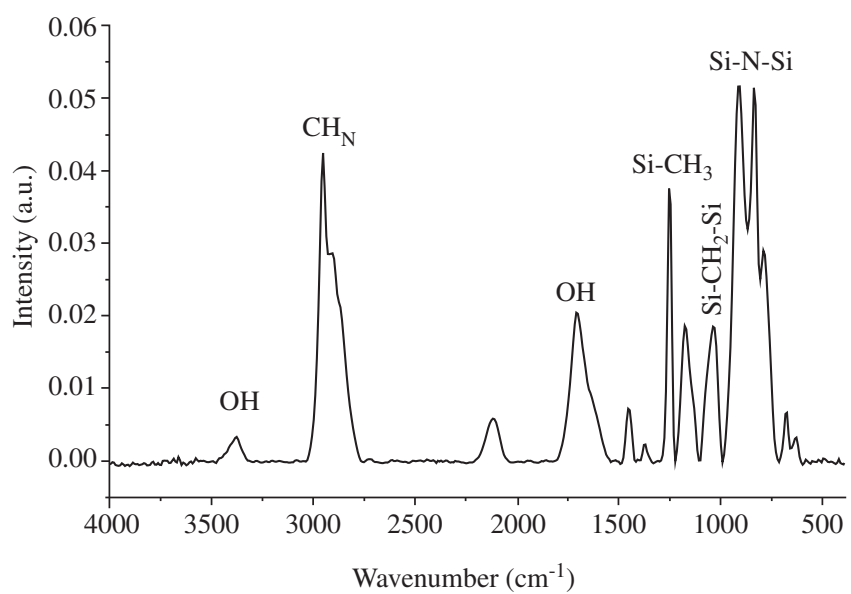

(b)

Figure 6. Typical FTIR spectra: a) HMDS ( 0.5 Torr, $250 \mathrm{~W}, 380 \AA$ A) and acetone (1.0 Torr, $250 \mathrm{~W}, 100 \AA$ ) double layer; and b) For HMDS (50 W; $500 \mathrm{mTorr}$; $1000 \AA$ ) and 2-propanol (150 W; 1.0 Torr; $3000 \AA$ ) double layer films obtained using a planar plasma reactor coupled with a $40 \mathrm{kHz}$ power supply.

Table 4. Results for island formation when double layer deposition is used.

\begin{tabular}{llccc}
\hline \multicolumn{1}{c}{ Properties } & & \multicolumn{2}{c}{ Reactants } \\
& Ethyl Ether/HMDS & 2-propanol/HMDS & Acetone/HMDS & 2-propanol/HMDS \\
\hline Islands formation & no & yes & no & no \\
Surface wrinkling & yes & no & no & no \\
System used & Planar $40 \mathrm{kHz}^{29}$ & Planar $40 \mathrm{kHz}^{31}$ & Planar $13.56 \mathrm{MHz}$ & Tubular $13.56 \mathrm{MHz}$ \\
\hline
\end{tabular}




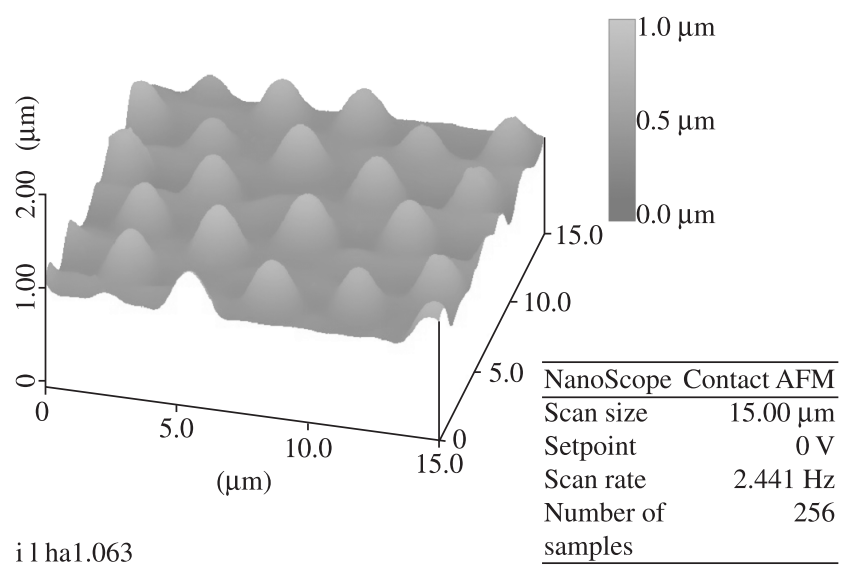

(a)

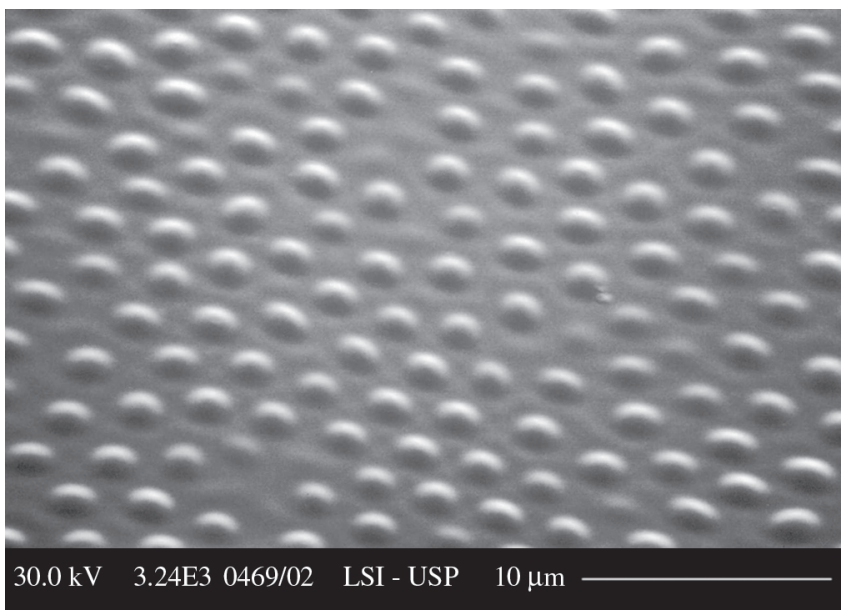

(b)

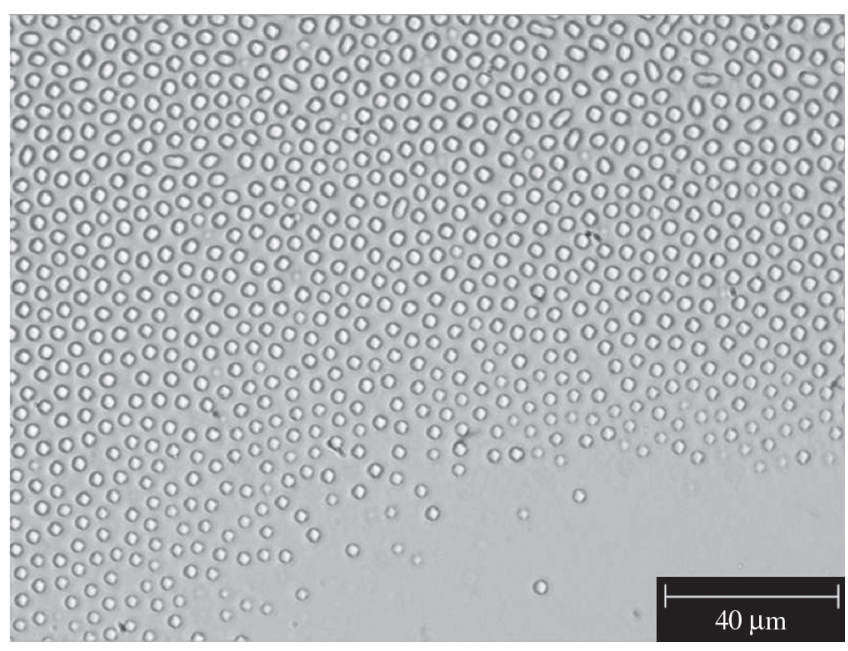

(c)

Figure 7. 2-propanol plasma (1.0 Torr, $150 \mathrm{~W}$ ) deposited on HMDS substrate (0.5 Torr, $50 \mathrm{~W}, 1000 \AA)$ using $40 \mathrm{kHz}$ as power supply planar reactor. Images obtained by: a) AFM; b) SEM; and c) Optical microscopy.

by plasma polymerization. For both reactors the use of $13.56 \mathrm{MHz}$ power supply, for all reagents, made it difficult to obtain films and usually the produced films are less polar. A possible explanation for

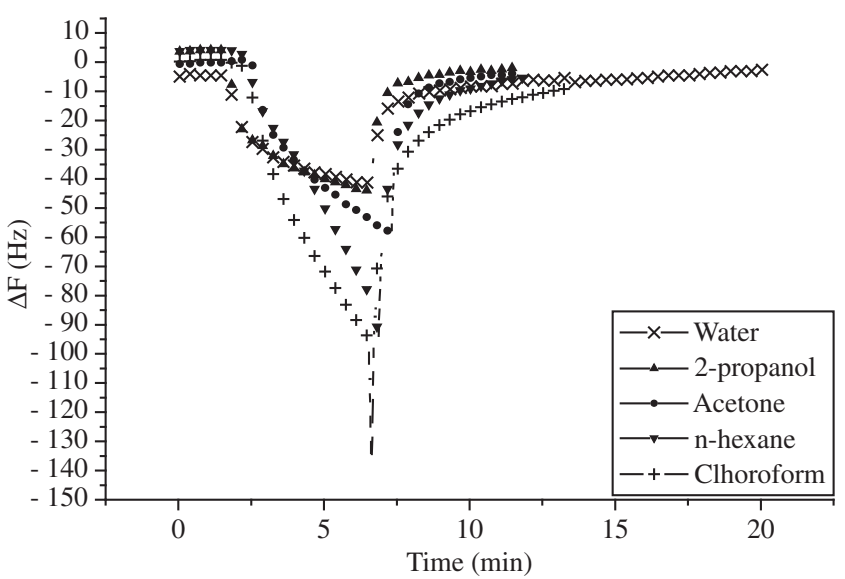

Figure 8. Frequency variation of $\mathrm{PQC}$ with a double layer film deposited on it.

this behavior is the different active species found at this frequency along with a lower surface bombardment during film formation.

At $40 \mathrm{kHz}$, the oxygen atom from the reagent molecule remains within the films and the deposition leads to polar films that do not easily deposit on hydrophobic surfaces, which results on islands or wrinkling. The active species at $13.56 \mathrm{MHz}$ probably lost the oxygen atom in elimination mechanisms and lead to polyethylene-like film formation that favors deposition on hydrophobic surfaces. Therefore, there is no island or wrinkling formation.

The double layer film can be useful for: 1) removal of small quantity of water due to the polar characteristic of the 2-propanol plasma film; and 2) preconcentration or removal of organic compounds due to the HMDS film adsorption characteristics. These applications are studied in the second part of this paper.

\subsection{Microstructures}

As mentioned in the Introduction, microchannels and cavities are important structures for preconcentrators or sensor development, especially when used with adsorbent films deposited on their surface.

In this session the use of a) cavities for testing the adsorption properties of the substrates, with or without adsorbent thin films deposited on it; b) channels with constrictions to evaluate the importance of turbulences on the adsorption of polar thin films; and c) microchannels and adsorbent films for preconcentration of polar organic compounds are discussed. These microchannels have two different geometries: planar and non-planar.

\subsubsection{Cavities}

Cellulose surface has already been treated with plasma polymerized HMDS or double layer films for obtaining selective membranes to aqueous solutions $\mathrm{s}^{37,38}$. Therefore, cellulose is a good candidate for preconcentration or removal of organic compounds from gas phase using double layer deposition. In this work, the cavities were used to test cellulose as a substrate for preconcentration or removal organic compounds from nitrogen.

The cavity without any substrate and cellulose without any treatment were tested as reference. The surface of the cellulose film was modified by plasma deposition of a double layer of 2-propanol/ HMDS films. The cellulose inserted in the cavities was tested using QCM measurements and reactants in a large range of polarity carried by nitrogen. All the measurements were performed admitting the contaminants inside the cavity during 10 minutes. The frequency variation $(\Delta \mathrm{F})$ obtained with film deposited on the cellulose inside 
the cavity was compared to the one obtained using the cavity without cellulose in it. Table 5 shows the reduction of $\Delta \mathrm{F}$ obtained using the cavity with cellulose modified by plasma polymerization. Cavities without any cellulose in it and the setup without any structure were also tested for all organic contaminants and it cannot be seen any difference between the values. Therefore, the cavity does not adsorb significantly and can be used as reference. Cellulose substrates showed a high amount of water permeation that caused a swell of the substrate, as expected. All modified cellulose showed reasonable adsorption for organic compounds, as can be seen from Table 5. The low value $(53 \%)$ obtained for $n$-hexane is most likely due to the low polarity of the reactant.

\subsubsection{Chicane}

Microchannels with constrictions ${ }^{30}$ may show retention of organic compounds carried by nitrogen due to the turbulent flow caused by constrictions. Therefore, to evaluate the importance of turbulences on the adsorption of the 2-propanol thin film (a polar film) a structure named chicane was manufactured. In order to avoid capillarity effects large dimensions were used.

The chicane was tested using QCM analysis and polar and nonpolar reactant (water and n-hexane), respectively, carried by nitrogen flow. All measurements were performed admitting the contaminants inside the cavity during 10 minutes. The frequency variation $(\Delta \mathrm{F})$ obtained with film deposited inside the chicane was compared to the values obtained without any structure, i.e. without turbulence or retention due to film deposition. Table 6 shows the reduction of $\Delta \mathrm{F}$ obtained when using the chicane modified by plasma polymerization with polar and non-polar reactants. The high value obtained for water is most likely due to adsorption on the film surface. Again, the small value obtained for n-hexane is probably due to the low polarity of the molecule. 2-propanol vapor was inserted after water exposure and without nitrogen purge. The reagent shows a small retention (22\%), which indicates that water adsorbs on the surface and makes the later 2-propanol adsorption difficult. Therefore, the adsorption on a polar film may occur even with constrictions (turbulent flow).

\subsubsection{Microchannels}

Microchannels in two different shapes were modified by plasma deposition. Three types of films were deposited on microchannels with spiral shapes a) HMDS plasma polymerized film; b) double layer plasma polymerized film; and c) 2-propanol plasma polymerized film. Non-planar microchannels with ethyl ether plasma polymerized film ${ }^{29}$ were also used. The ethyl ether film is useful for adsorption of organic compounds, however it is less polar than the 2-propanol ones. Therefore some difference may occur during preconcentration phenomena.

The tests were carried out using the setup described in Materials and Methods for liquid phase. The reactants (analyte) were injected in less than 10 seconds, in a single pulse of $0.05 \mathrm{~mL}$, for all cases. Therefore the experiment is similar to an analysis using a chromatographic system. The non-planar structure, with or without ethyl ether film, was tested only for 2-propanol.

Table 7 shows the frequency variation $(\Delta \mathrm{F})$ obtained by injecting reactants with spiral shape. The non-planar structure shows a frequency variation of $100 \mathrm{~Hz}$ and $95 \mathrm{~Hz}$ with or without ethyl ether film, respectively, when tested for 2-propanol. It can be seen from Table 7 that the non-polar reactant (n-hexane) is not adsorbed on double layer films due to the presence of 2-propanol plasma films on the surface, which favors the adsorption of more polar compounds. For acetophenone, the adsorption easily occurs on HMDS plasma film due to the phenyl group of the molecule. The adsorption of acetone and 2-propanol on HMDS and 2-propanol plasma films is in good agree-
Table 5. Reduction of $\Delta \mathrm{F}(\%)$ obtained using the cavity for thin film deposited on cellulose. Cavity without films deposited in it was used as reference.

\begin{tabular}{cc}
\hline $\begin{array}{c}\text { Reactant Carried } \\
\text { by } \mathrm{N}_{2}\end{array}$ & $\begin{array}{c}\Delta \text { F Reduction for Cellulose with Double } \\
\text { Layer Deposition on Cavities (\%) }\end{array}$ \\
\hline n-hexane & 53 \\
Acetone & 93 \\
2-propanol & 91 \\
Water & 75 \\
\hline
\end{tabular}

Table 6. Reduction of $\Delta \mathrm{F}$ on percentages obtained using the chicane with 2-propanol plasma film deposited on it. Setup without chicane it was used as reference.

\begin{tabular}{cc}
\hline Reactant Carried by $\mathrm{N}_{2}$ & $\Delta$ F Reduction $(\%)$ \\
\hline n-hexane & 20 \\
water & 82 \\
2-propanol* & 22 \\
\hline
\end{tabular}

*used after water

Table 7. Frequency variation $(\Delta \mathrm{F}-\mathrm{Hz})$ obtained on the detector after injection of reactants in spiral shape microchannels.

Plasma Polymerized Reactants

Film Deposited in n-hexane Acetophenone Acetone 2-propanol the Channels

\begin{tabular}{lrccc}
\hline Without film & 110 & 1800 & 22 & 170 \\
HMDS & 70 & 140 & 13 & 50 \\
Double layer & 125 & - & - & - \\
2-propanol & - & - & 2 & 95 \\
\hline
\end{tabular}

ment with the results observed by QCM measurements ${ }^{31,32}$ in gaseous phase. Therefore, these films are useful for sample preconcentration of organic compounds in both phases: gas and liquid.

\section{Conclusions}

Double layers films are promising for adsorption of water in gas phase, as shown by QCM measurements. The films can be also useful for retention of organic compounds in liquid phase, as shown by the deposition on microchannels. The 2-propanol plasma films can be also used for retention, even in turbulent flow, as verified using a chicane structure.

The results also point out that cellulose is a promising substrate for low cost and disposable preconcentration devices, especially on gas phase.

The retention capacity of covered microchannels was, in general, higher than non-modified ones. Therefore, deposition of polar or double layers plasma films in microchannels favors adsorption. These results point out that the use of microchannels modified by 2-propanol or double layers plasma films can be useful for preconcentration of polar organic compounds, in gaseous or liquid mixtures.

\section{Acknowledgments}

To FAPESP and CNPQ for financial support.

\section{References}

1. Lichtenberg J, Rooji NF, Verpoorte E. Sample pretreatment on microfabricated devices, Talanta. 2002; 56:233-266. 
2. Mamginell RP, Rosato DA, Benson DA, Frye-Mason GC. Finite Element Modeling of a Microhotplate for Microfluidic. Applications, MEMs 99, San Juan: Puerto Rico; 1999.

3. Albuquerque NM. Micro Analytical Systems Department Technology$\mu$ ChemLabTM Fact Sheet; $\mu$ ChemLab Technology Team, Autonomous Micro-Chemical Analysis Laboratory ( $\mu$ ChemLab Technologies). Sandia Report; 2001 Jun; 1997.

4. Lindner $D$, The $\mu$ ChemLabTM project: micro total analysis system $R \& D$ at Sandia National Laboratories, Lab on a Chip 1. 2001; 1:15N-19N.

5. Sylwester A. Sandia's $\mu$ ChemLab $\mu$ Program. CPAC Summer Institute 2001 Proceedings and Summary Topics in Process Analysis and Control with special emphasis on: Developing Analytical Tools for High Throughput Experimentation. CA: USA, 2001 Jul; p. 25-27.

6. Ho CK, Wright J, McGrath LK, Lindgren ER, Rawlinson KS, Lohrstorfer CF. Field Demonstrations of Chemiresistor and Surface Acoustic Wave Microchemical Sensors at the Nevada Test Site. Sand Report. 2003 Mar, Sand 2003-0799: p. 3-77.

7. Ho CK, Lohrstorfer CF. In situ monitoring of vapor phase TCE using a chemiresistor microchemical sensor, $\mathrm{CF}$ ground Water Monitoring and Remediation. FAL. 2003; 23(4):85-90.

8. Ho CK, Lindgren ER, Rawlinson KS, McGrath LK. Wright Development of a Surface Acoustic Wave Sensor for In-Situ Monitoring of Volatile Organic Compounds. JL SENSORS. 2003 Jul; 3(7):236-247.

9. Rivera D, Alam MK, Davis CE, Ho CK. Characterization of the Ability of Polymeric Chemiresistor Arrays to Quantitate Trichloroethylene Using Partial Least Squares (PLS): Effects of Experimental Design, Humidity, and Temperature. Sensors \& Actuators Chemical B. $2003 \mathrm{Jul}$; 92(1-2): 110-120.

10. Ho CK, Hughes RC. In-situ Chemiresistor Sensor Package for Real-time detection of Volatile Organic Compounds in Soil and Groundwater. Sensors. 2002 Jan; 2(1):23-34.

11. Center for Environmental Research Information Office of Research and Development U.S. Environmental Protection Agency. Compendium of Methods for the Determination of Toxic Organic Compounds in Ambient Air, Second Edition - Compendium Method TO-15 - Determination of Volatile Organic Compounds (VOCs) in Air Collected In Specially-Prepared Canisters and Analyzed by Gas Chromatography/ Mass Spectrometry (GC/MS), EPA/625/R-96/010b, Cincinnati; January 1999.

12. Feng $\mathrm{CH}$, Mitra S. Two-stage Microtrap as an Injection Device for Continuous On-Line Gas Chromatographic Monitoring. Journal of Chromatography. 1998 May; A 805(1-2):169-176.

13. Mitra S, Xu YH, Chen WJ, Lai A. Characteristics of Microtrap-based Injection Systems for Continuous Monitoring of Volatile Organic Compounds by Gas Chromatography. Journal of Chromatography. 1996 Mar; A 727(1):111-118.

14. Mitra S, Zhu NH, Zhang X, Kebbekus B. Continuous Monitoring of Volatile Organic Compounds in Air Emissions Using an On-Line Membrane Extraction-microtrap-gas Chromatographic System. Journal of Chromatography. 1996 Jun; A 736(1-2):165-173.

15. Kim A, Mitra S. A Microfabricated Microconcentrator for Sensors and Gas Chromatography. Journal of Chromatography. 2003 May; A 996(1-2):1-11

16. Tian C, Pang W, Lu CJ, Zellers ET. Microfabricated Preconcentrator/focuser for a Microscale Gas Chromatograph. J. Microelectromechanical Systems. 2003; 12(3):264-23.

17. Tian WC, Pang SW. Performance of Preconcentrator/Focuser with Microfabricated Si Heater, University of Michigan; 2002. Available from http://www.eecs.umich.edu/ pang/projects.

18. Tian WC, Pang SW. Thick and Thermally Isolated Microheaters for Preconcentrator/Focuser in Micro Gas Chromatography University of Michigan; 2002. Available from: http://www.eecs.umich.edu/ pang/ projects.
19. Tian WC, Pang SW. Freestanding Microheaters in Si with High Aspect Ratio Microstructures". J Vac Sci Tech B. 2002; 20:1008-1018.

20. Tian WC. Design, Fabrication and Characterization of Microfabricated Preconcentrator-focuser for Micro Gas Chromatography. [Thesis]. University of Michigan; 2003.

21. Nakamoto T, Isaka Y, Ishige T, Moriizumi T. Odor-sensing System Using Preconcentrator With Variable Temperature. Sensors \& Actuators Chemical-B. 2000 Sep; 69(1-2):58-62.

22. Nakamoto T, Sumitimo E. Study of Robust Odor Sensing System With Auto-sensitivity Control. Sensors \& Actuators Chemical B. 2003 Apr; 89(3):285-291.

23. Czae MZ, Wang J. Pushing the Detectability of Voltammetry: How Low Can We Go? Talanta. 1999 Dec; 50(5):921-928.

24. Uchiyama K, Nakajima H, Hobo T. Detection Method for Microchip Separations. Analytical and Bioanalytical Chemistry. 2004 Jun; 379(3): 375-382.

25. Silva JAF, Furlan R, Simões EW, Silva MLP, Pereira MT. Simulations of Silicon Microstructure Preconcentration of Metallic Ions. $8^{\text {th }}$ Symposium on Microelectronics Technology and Devices. Porto Alegre: Brasil; 2003.

26. Huber DL, Manginelli RP, Samara MA, Kim BI, Bunker BC, Programmed Adsorption and Release of Proteins in a Microfluidic Device. Science. $2003 ; 5631: 352-354$

27. Guo XM, Mitra S. On-line Membrane Extraction Liquid Chromatography for Monitoring Semi-volatile Organics in Aqueous Matrices. J Chromatography A. 2002; 904(2):1189-1196.

28. Lago CL do, da Silva HDT, Neves CA, Brito-Neto JGA, da Silva JAF. A dry process for production of microfluidic devices based on the lamination of laser-printed polyester films. Analytical Chemistry. 2003 Aug; 75(15): 3853-3858.

29. Lima RR, Carvalho RAM, Nascimento Filho AP, Silva MLP, Demarquette N R. Production of Adsorbent Films and Deposition on Low Cost Micromachined Non-Planar Microchannels for Preconcentration of Organic Compound in Air. Chemical Sensors. 2004; 20(B):604

30. Souza SG. Estudo da Viabilidade Técnica da Utilização de Microcanais para Concentração de Produtos Químicos. [Thesis]. EPUSP, Brazil; 2003.

31. Nascimento AP, Silva MLP, Galeazzo E, Demarquette NR. Use of plasma polymerized highly polar organic compound films for sensor development. Sensors \& Actuators. 2003; B 91(1-3):370-377.

32. Silva MLP, Tan IH, Nascimento AP, Galeazzo E, Jesus DP. Use of plasma polymerized highly hydrophobic hexamethyldissilazane (HMDS) films for sensor development. Sensors \& Actuators. 2003; B91(1-3):362-369.

33. Carvalho AT, Silva MLP, Santos Filho SG, Nascimento Filho AP, Jesus DP. Improvement on organic compound adsorption and or detection by using metallic thin films deposited onto righly-rough silicon substrates. Chemical Sensors. 2004; 20(B):572.

34. Tan IH, Demarquette NR, Silva MLP, Degasperi FT, Dallacqua R. Adhesion improvement of cellulose to polypropylene: a comparison of plasma treatment o PP and plasma deposition on cellulose. ISPC-14 Symposium Proceedings. Prague: Czech Republic; 1999. p. 1907-1917.

35. Matos RC, Gutz IGR, Angnes L, Fontenele RS, Pedrotti JJ. Propulsor Pneumático Versátil e Isento de Pulsação para Sistemas de Análise Em Fluxo. Química Nova. 2001; 24(6): 795-798.

36. Santos AD, Carvalho T, Silva MLP. Proposta de sistema miniaturizado para ensino em engenharia química. XV COBEQ. Curitiba, Brasil; 2004 Sep.

37. Silva MLP, Demarquette NR, Tan IH. Use of HMDS/Hexane double layers for obtaining low cost selective membrane. Cellulose. 2003; 10: 171-178.

38. Tan IH, Silva MP, Demarquette NR. Paper surface modification by plasma deposition of double layers of organic silicon compounds. J Mater Chem. 2001; 11:1019-1025. 